\title{
Accuracy of the SpineNav3DTM Technology to Measure the Depth of Epidural Space: A Comparison with the Standard Ultrasound Technique in Pregnant Volunteers
}

\author{
Giorgio Capogna $^{1 *}$, Simone Baglioni'2, Valentina Milazzo ${ }^{3}$, Arturo Vitale ${ }^{4}$ \\ ${ }^{1}$ Department of Anesthesiology, Città di Roma Hospital, Roma, Italy \\ ${ }^{2}$ Department of Anesthesiology, ASST S. Gerardo-Monza, Monza, Italy \\ ${ }^{3}$ Department of Anesthesiology, University of Catania, Catania, Italy \\ ${ }^{4}$ Statistical Consultant, Roma, Italy \\ Email: *capogna.giorgio@gmail.com
}

How to cite this paper: Capogna, G. Baglioni, S., Milazzo, V. and Vitale, A. (2018) Accuracy of the SpineNav3DTM Technology to Measure the Depth of Epidural Space: A Comparison with the Standard Ultrasound Technique in Pregnant Volunteers. Open Journal of Anesthesiolo$g y, 8,113-122$.

https://doi.org/10.4236/ojanes.2018.84012

Received: March 1, 2018

Accepted: April 17, 2018

Published: April 20, 2018

Copyright (c) 2018 by authors and Scientific Research Publishing Inc. This work is licensed under the Creative Commons Attribution International License (CC BY 4.0).

http://creativecommons.org/licenses/by/4.0/

(c) (i) Open Access

\begin{abstract}
Background: The SpineNav3DTM technology was recently incorporated in the Accuro device for the automatic detection of spinal bone landmarks. The goal of our study was to validate the ability of the Accuro ultrasound scanner to detect the distance from skin to epidural space by comparing it to the golden standard (the standard ultrasound). The secondary end-point was the inter-rater agreement between an expert anesthesiologist and a novice trainee in determining the epidural space depth with the Accuro device. Methods: 96 consecutive healthy volunteer parturients at term had their lumbar area scanned for the measurements of the depth of the epidural space (recorded in $\mathrm{cm}$ ) by two anesthesiologists. The expert investigator made the measurements with both the standard ultrasound and the Accuro devices, and the trainee made the measurements with the Accuro device only. Results: There were no differences in the mean depth of the epidural space as measured by the expert or the novice investigator with both the devices (standard ultrasound and Accuro) in both positions (sitting and lateral) at any intervertebral level with a difference less than $0.25 \mathrm{~cm}$. Conclusion: We have demonstrated that this handheld ultrasound system with 3D spine navigation technology can automatically identify the key neuraxial landmarks within ultrasound images of the lumbar spine acquired in the transverse plane and measure the epidural depth with the same accuracy as the standard ultrasounds. We have also demonstrated that the measurements may be rapidly and adequately obtained by a novice who had never previously used ultrasounds.
\end{abstract}




\section{Keywords}

Ultrasound, Epidural, Obstetrics

\section{Introduction}

The most commonly used ultrasound procedure to identify intervertebral spaces is the pre-puncture technique, which has been shown to be helpful in achieving correct epidural placement, especially for teaching purposes and in the obese parturient [1] [2] [3] [4] [5]. However, evidence on ultrasound-guided identification of the epidural space in pregnancy is still limited and this technique is not commonly used as a routine tool to detect the epidural space in obstetrics since the visibility of the ligamentum flavum, of the dura mater and of the epidural space decrease significantly during pregnancy [6].

In addition to financial constraints, the low availability of a dedicated ultrasound machine and the perceived poor evidence of its benefits, means that the routine use of ultrasounds for the identification of the epidural space is still heavily dependent on the user's familiarity and skill with ultrasonography [7].

The SpineNav3DTM technology has been recently developed to facilitate image interpretation of individual 2D lumbar spine scans by automating spinal bone landmark detection and depth measurements and providing a real-time assessment of scan plane orientation in 3D in a pocket-sized, battery operated ultrasound instrument, the Accuro [8].

This technology is able to provide some basic information such as detecting the bone landmarks and measuring the depth of the epidural space and was able, in a preliminary report, to accurately predict the Touhy needle depth to epidural space [9].

We decided to undertake this study to evaluate the concordance of the values provided by the Accuro with those obtained by a standard ultrasound machine.

The primary aim of our study was to test the accuracy of the Accuro device in determining the depth of the epidural space by adopting a midline approach using as comparator the measurement obtained with the standard ultrasound device by an expert operator. The secondary end-point was the inter-rater agreement, or concordance, between an expert anesthesiologist and a novice trainee who had never used an ultrasound device to determine the epidural space depth.

\section{Methods}

The local ethics board approved the study which was registered at Clinical Trials. gov with the identifier NCT03164369. The study population consisted of 96 consecutive healthy parturients at term, who volunteered and gave their written informed consent to be investigated with lumbar ultrasounds during their antenatal anesthetic consultation.

This prospective, semi blinded study was conducted at the department of Anes- 
thesia of the Città di Roma Hospital, Roma in May 2017.

All the ultrasound scans were conducted by the same two investigators.

This manuscript adheres to the applicable Equator Guidelines and the study followed the Guidelines for the safe use of diagnostic ultrasound equipment (ALARA).

For the purpose of the study we used an Accuro ${ }^{\circledR}$ device (Rivanna Medical, LLC, Charlottesville, VA - USA) which incorporates a $5 \mathrm{MHz}$ probe, and two portable ultrasound machines in B-Mode: GE Logiq Book XP ${ }^{\circledast}$ (Soma Technology Inc., Bloomfield, CT - USA) and My Lab 25 (Esaote, Genova-Italia) using two 2 - $5.5 \mathrm{MHz}$ convex probes.

All the subjects had their lumbar area scanned for the measurements of the depth of the epidural space (recorded in $\mathrm{cm}$ ) defined as the distance from the skin to the posterior complex (ligamentum flavum, epidural space, and posterior dura) by two anesthesiologists, one expert in ultrasound and the other a novice,a trainee in anesthesia, who had never used an ultrasound machine or been previously instructed in the use of the Accuro device.

In order to identify the intervertebral levels, the expert investigator, using the standard ultrasounds, moved the proble cephalad to obtain the classic hyperechoic saw-like image representing the articular processes, and counted the interspaces upward till the desired interspace. At this time, the study started using the transverse scan.

All parturients were examined in both the sitting and the lateral position at L2-L3 and L3-L4 intervertebral levels previously identified by the expert investigator.

The expert investigator made the measurements with both the standard ultrasound and the Accuro devices, the trainee made the measurements with the Accuro device only.

Both the investigators were blinded to the other's measurements.

The midline was identified with the standard ultrasound machine, by a convex probe $2-5 \mathrm{MHz}$, to identify the L2-L3 and L3-L4 interspaces and to obtain the view of the complex formed by the ligamentum flavum-dura mater allowing the measurement of the depth of the epidural space.

With the Accuro device the midline was obtained by moving the device left or right and/or upwards and downwards to ensure the mid-line indicator was centered on the imaging screen. Thereafter the device was moved along the lumbar region until the interlaminar space and the epidural depth were automatically identified and measured at the desired intervertebral level (Figure 1 and Figure 2).

\section{Power Calculation}

Assuming a Type I error rate of $5 \%(\alpha=0.05)$ and a Type II error rate of $20 \%$ ( $\beta$ $=0.2$ ), a minimum of 76 subjects were required for this study to detect a maximum difference of $0.25 \mathrm{~cm}$ in the measurement of the distance between the skin 


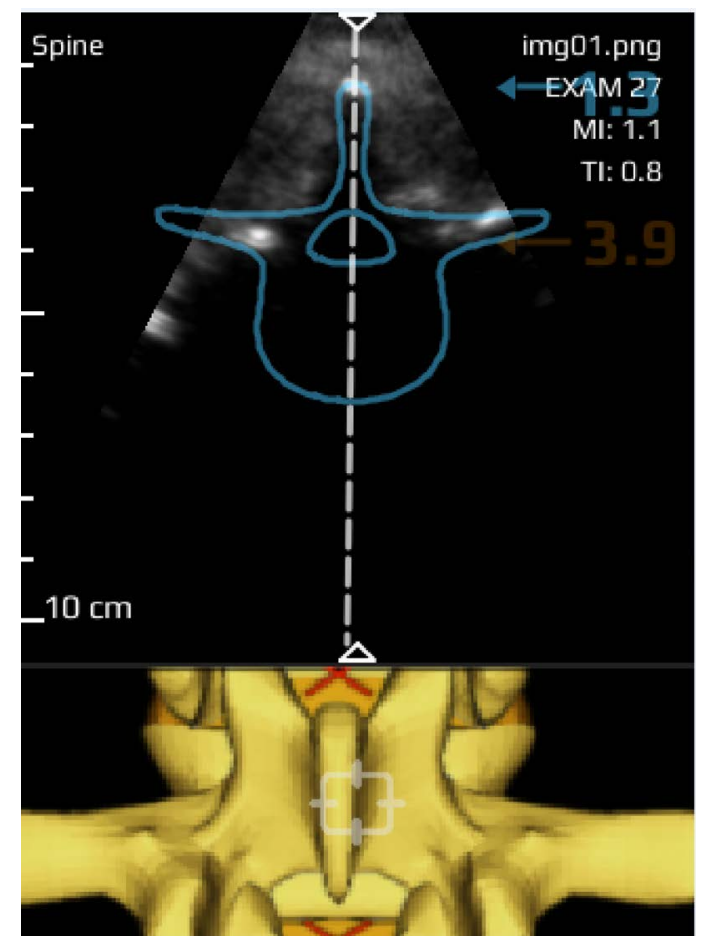

Figure 1. Graphics rendered to the Accuro screen by the CAD algorithm to indicate that the image contains a spinous process. Also shown are depth and midline indicators, along with a bird's-eye view of the 3D spine model. The blue number is the depth to the spinous process tip (in centimeter).

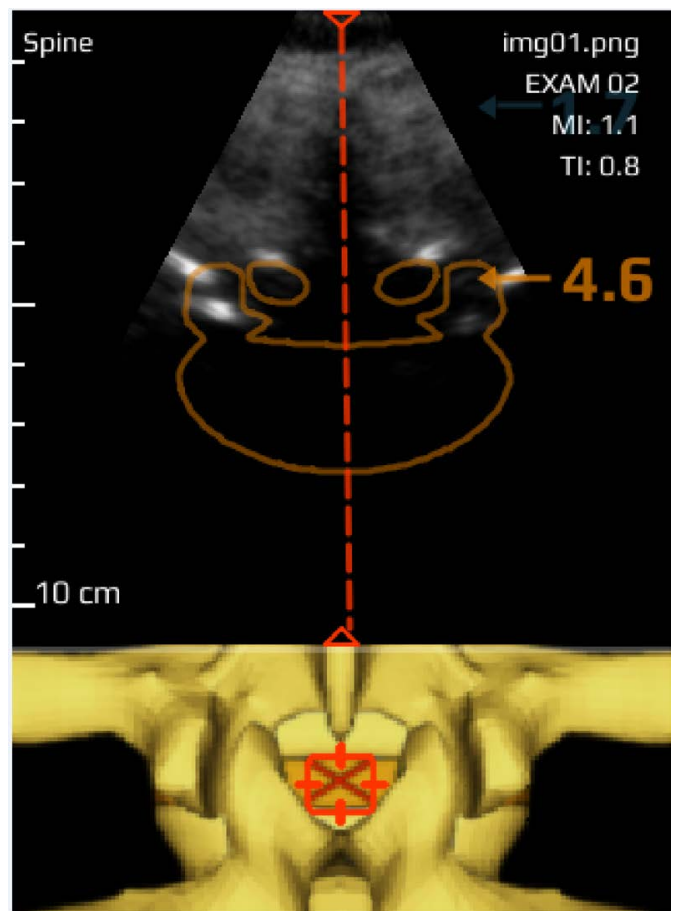

Figure 2. Graphics rendered to the Accuro screen by the CAD algorithm to indicate that the image contains an epidural space. Also shown are depth and midline indicators, along with a bird's-eye view of the 3D spine model. The orange number is the depth to the epidural space (in centimeter). 
and the epidural space between the two devices (standard ultrasound and Accuro).

\section{Statistical Analysis}

Normality tests, Shapiro-Wilk and Lilliefors (Kolmogorov-Smirnov), were used to check if the variables were normally distributed. The T-test was used to compare standard ultrasound scanner measurements with those of the Accuro ultrasound scanner and the difference between the measurements made with the Accuro by the expert and the beginner both in the sitting and lateral position.

The Bland-Altman plot was used to compare the standard ultrasound vs Accuro and the expert's vs the novice's measurements performed with the Accuro device.

\section{Results}

All the subjects completed the study. Mean age was $33.01 \pm 5.51$ years, mean gestational weight was $72.67 \pm 12.51 \mathrm{Kg}$, mean height was $163 \pm 6 \mathrm{~cm}$, mean gestational BMI was $27.18 \pm 4.35$ and mean gestational age was $35.6 \pm 2.6$ weeks. All the patients were pimigravidae.

Results are reported in Table 1. There were no differences in the mean depth of the epidural space as measured by the expert or the novice investigator with both the devices (standard ultrasound and Accuro) in both positions (sitting and lateral) at any intervertebral level. There were no differences between the expert's and the novice's measurements.

In the Figure 3, Figure 4 the Bland Altman curves are reported that compare the overall results obtained by the novice and the expert investigator and the results obtained by the expert investigator with the standard ultrasound and the Accuro measurements. Horizontal lines are drawn at the mean difference and at the limits of agreement, which are defined as the Means Difference plus and minus 1.96 times the standard deviation of the differences.

In all the graphs the mean is very close to $0.25 \mathrm{~cm}$ which was our pre-determined clinical difference end point.

\section{Discussion}

The use of preprocedural ultrasound has been extensively studied as a tool to

Table 1. Depth of epidural space $(\mathrm{cm})$. NS = not significant difference.

\begin{tabular}{ccccccccccccc}
\hline & \multicolumn{3}{c}{} & \multicolumn{3}{c}{ Accuro } & \multicolumn{3}{c}{ Standard Ultrasound } \\
\hline & \multicolumn{3}{c}{} & \multicolumn{3}{c}{ Expert } & \multicolumn{3}{c}{ Beginner } & \multicolumn{4}{c}{ Expert } \\
\hline \multirow{2}{*}{ lateral } & Mean & S.D. & C.I. (95\%) & Mean & S.D. & C.I. (95\%) & Mean & S.D. & C.I. (95\%) & NS \\
\hline \multirow{3}{*}{ seated } & L2-L3 & 4.4 & 0.76 & $4.32-4.47$ & 4.3 & 0.72 & $4.22-4.37$ & 4.8 & 0.61 & $4.70-4.83$ & NS \\
& L3-L4 & 4.7 & 0.76 & $4.62-4.77$ & 4.6 & 0.76 & $4.52-4.67$ & 5.0 & 0.67 & $4.93-5.06$ & NS \\
& L2-L3 & 4.4 & 0.60 & $4.33-4.46$ & 4.5 & 0.62 & $4.43-4.56$ & 4.7 & 0.77 & $4.65-4.80$ & NS \\
& L3-L4 & 4.8 & 0.64 & $4.73-4.86$ & 4.8 & 0.67 & $4.73-4.86$ & 4.9 & 0.77 & $4.80-4.95$ & NS \\
\hline
\end{tabular}




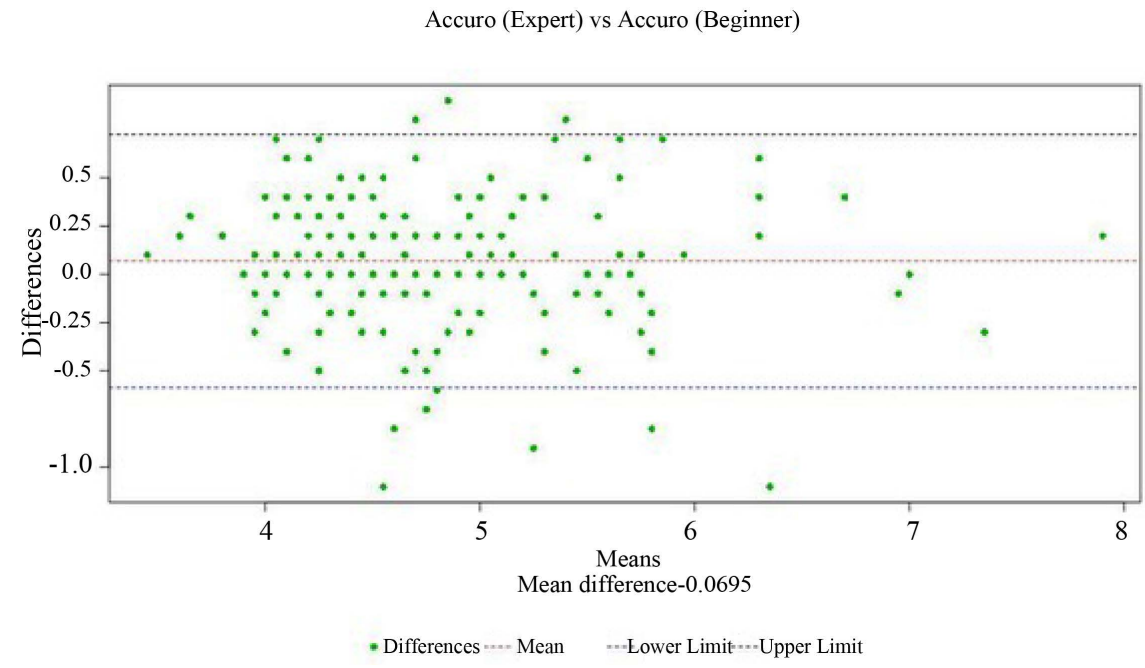

Figure 3. Bland Altman curve that compare the overall results obtained by the novice and the expert investigator with the Accuro measurements (depth of epidural space).

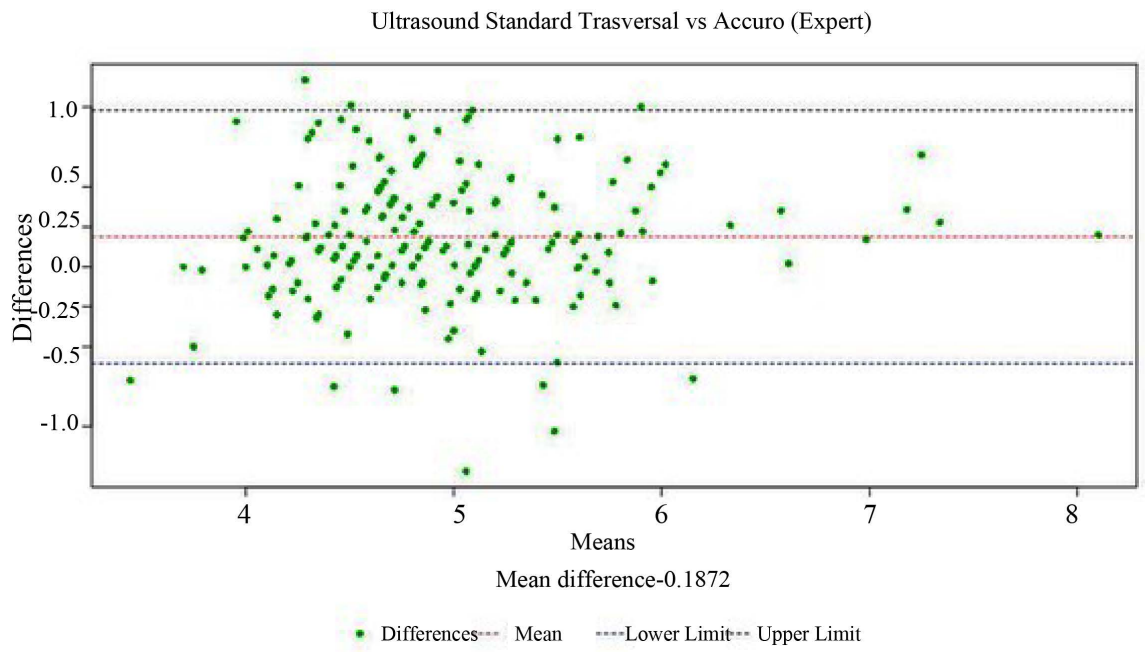

Figure 4. Bland Altman curve that compare the overall results obtained by expert investigator with the standard Ultrasound and the Accuro measurements (depth of epidural space).

improve epidural success rates in difficult patients by providing an additional tool to identify key neuraxial landmarks before needle insertion [10] [11] [12]. When associated to palpation-based methods, preprocedural ultrasound improves first-attempt epidural success rates, reduces the number of needle passes, and accurately estimates the depth to the epidural space within approximatelylcm [13] [14]. However, the evidence suggests that the acquisition and retention of neuraxial ultrasonography skills represents a major impediment to the widespread use of ultrasound for neuraxial bedsides procedures [7].

In addition, individuals performing the ultrasound imaging were study investigators who generally had high levels of experience and special interest in the field [15]. 
Accuro is a pocket-sized, battery operated device consisting of an ultrasound system, ultrasound probe, and rotatable touch-screen display. It incorporates the SpineNav3D technology, to automate spinal bone landmark detection and depth measurements and to assess real-time scan plane orientation in 3D.

In a previous study Tiouririne et al. [16] established the agreement between automated anatomical landmark detection of the lumbar spine by Accuro algorithm and manual landmark identification with a portable V-Scan performed by experienced radiologists .

Accuro detected the epidural space with a sensitivity of $94.2 \%$ (95\% confidence interval [CI], $85.1 \%-98.1 \%)$ and a specificity of $85.5 \%$ (95\% CI, $81.7 \%$ $88.6 \%)$ and measured its depth with an error of approximately $\pm 0.5 \mathrm{~cm}$ compared to measurements obtained manually from the 2-dimensional ultrasound images.

Our results indicated that the measurements of the epidural depth obtained by an expert with a standard ultrasound machine are comparable to those obtained by the Accuro, at any intervertebral level either in the sitting or in the lateral position with a difference less than $0.25 \mathrm{~cm}$.

These precision differences in accuracy might be due to different methods of collecting the data. In the study of Tiouririne, performed on a non homogeneous group of volunteers, the cinevideo ultrasound data set recorded by an anesthesiologist expert in ultrasound who used a portable V-scan machine was examined by three independent radiologists. In our study a single expert physician made all the measurements on a homogeneous group of pregnant women at term.

We believe this degree of accuracy is considered to be adequate for determining where to place the needle along the spine midline and for providing an estimate of the depth to the epidural space.

The primary impediment to widespread adoption of preprocedural ultrasonography for bedside epidural procedures appears to be its steep learning curve. Deacon et al. [17] and Margarido et al. [18] determined that fewer than 40\%of trainees were able to identify an appropriate needle insertion location on a subject with normal BMI after comprehensive training by a team of experts. Image interpretation is even more difficult in obese and elderly patients due to degraded image quality, and key landmarks are only detectable by experts in $70 \%$ to $80 \%$ of obese patients [5].

Evidence suggests that ultrasound imaging of the spine by an experienced operator can achieve an accuracy rate of $>90 \%$ compared to $32 \%$ for novices [19].

The Accuro device has been introduced as a bedside, ready to use piece of equipment needing no prior expertise in ultrasounds. In our study we demonstrated that a novice with no experience in ultrasound may obtain, with the Accuro device, the same accuracy and reliability when determining the epidural depth as that obtained by a blinded expert investigator.

The goal of our study was to validate the ability of the Accuro ultrasound 
scanner to detect the distance from skin to epidural space by comparing it to the golden standard (the standard ultrasound) and not to evaluate or to compare two such different technologies. The Accuro device is designed to visualize bone landmarks and provides only an automatic epidural location and depth and therefore cannot be compared to and used as an ultrasound machine but as a "pointer".

This study is not without limitations. Although the Accuro ultrasound system can acquire ultrasound images in any orientation, the lumbar spine algorithm only assists with anatomical landmark recognition when imaging in the transverse plane. There is evidence that transverse plane imaging is sufficient for identifying the midline and depth to the epidural space [5] but imaging in longitudinal and sagittal orientations has also been shown to improve efficacy [20] [21]. In addition, the deepest epidural space observed in our study was less than $5 \mathrm{~cm}$ deep, and it is not representative of the most difficult patients, such as the obese.

Our study was performed with healthy term parturients during their antenatal visit and not on obese parturients, therefore no conclusion may be drawn from our results as far as this type of more challenging patients is concerned.

Given that this was an imaging-only study in volunteers, the reported depths measured from the ultrasound images should be validated by further studies to evaluate the true depths confirmed by needle placement.

\section{Conclusion}

In conclusion, we have demonstrated that this handheld ultrasound system with 3D spine navigation technology can automatically identify the key neuraxial landmarks within ultrasound images of the lumbar spine acquired in the transverse plane and measure the epidural depth with the same accuracy as the standard ultrasounds. We have also demonstrated that the measurements may be rapidly and adequately obtained by a novice who had never used ultrasounds previously.

\section{References}

[1] Grau, T., Bartusseck, E., Conradi, R., et al. (2003) Ultrasound Imaging Improves Learning Curves in Obstetric Epidural Anesthesia: A Preliminary Study. Canadian Journal of Anesthesia, 50, 1047-1050. https://doi.org/10.1007/BF03018371

[2] Grau, T., Leipold, R., Fatehi, S., Martin, E. and Motsch, J. (2004) Real-Timeultrasonic Observation of Combined Spinal-Epidural Anaesthesia. European Journal of Anaesthesiology, 21, 25-31.

[3] Grau, T., Leipold, R., Conradi, R., Martin, E. and Motsch, J. (2002) Efficacy of Ultrasound Imaging in Obstetric Epidural Anesthesia. Journal of Clinical Anesthesia, 14, 169-175. https://doi.org/10.1016/S0952-8180(01)00378-6

[4] NICE: National Institute for Health and Clinical Excellence. Ultrasound Guided Catheterization of the Epidural Space: Under-Standing NICE Guidance, 2008. https://www.nice.org.uk/guidance/ipg249/chapter/2-The-procedure 
[5] Balki, M., Lee, M., Halpern, S., et al. (2009) Ultrasound Imaging of the Lumbar Spine in the Transverse Plane: The Correlation between Estimated and Actual Depth to the Epidural Space in Obese Parturients. Anesthesia \& Analgesia, 108, 1876-1881. https://doi.org/10.1213/ane.0b013e3181a323f6

[6] Grau, T., Leipold, R.W., Horter, J., et al. (2001) The Lumbar Epidural Space in Pregnancy: Visualization by Ultrasonography. British Journal of Anaesthesia, 86, 798-804. https://doi.org/10.1093/bja/86.6.798

[7] Clegg, I., Bhatia, K., Kochhar, P. and Maguire, S. (2014) The Availability and Use of Ultrasound in Obstetric Anaesthesia in the UK. International Journal of Obstetric Anesthesia, 23, S17.

[8] Mauldin, F.W., Owen, K., Tiouririne, M., et al. (2012) The Effects of Transducer Geometry Onartifacts Common to Diagnostic Bone Imaging with Conventional Medical Ultrasound. IEEE Transactions on Ultrasonics, Ferroelectrics, and Frequency Control, 59, 1101-1114. https://doi.org/10.1109/TUFFC.2012.2301

[9] Seligman, K., Weiniger, C. and Carvhalo, B. (2017) Accuracy of a Handheld Ultrasound Device and a Traditional Ultrasound for Neuraxial Depth and Landmark Assessment in Cohort Receiving Labor Epidurals. SOAP 49 th Annual Meeting, Bellevue, May.

[10] Chin, K.J., Perlas, A., Chan, V., et al. (2011) Ultrasound Imaging Facilitates Spinal Anesthesia in Adults with Difficult Surface Anatomic Landmarks. Anesthesiology, 115, 94-101. https://doi.org/10.1097/ALN.0b013e31821a8ad4

[11] Arzola, C., Davies, S., Rofaeel, A., et al. (2007) Ultrasound Using the Transverse Approach to the Lumbar Spine Provides Reliable Landmarks for Labor Epidurals. Anesthesia \& Analgesia, 104, 1188-1192. https://doi.org/10.1213/01.ane.0000250912.66057.41

[12] Soni, N.J., Franco-Sadud, R., Schnobrich, D., et al. (2016) Ultrasound Guidance for Lumbar Puncture. Neurology Clinical Practice, 6, 358-368. https://doi.org/10.1212/CPJ.0000000000000265

[13] Perlas, A., Chaparro, L.E. and Chin, K.J. (2016) Lumbar Neuraxial Ultrasound for Spinal and Epidural Anesthesia: A Systematic Review and Meta-Analysis. Regional Anesthesia and Pain Medicine, 41, 251-260. https://doi.org/10.1097/AAP.0000000000000184

[14] Shaikh, F., Brzezinski, J., Alexander, S., et al. (2013) Ultrasound Imaging for Lumbar Puncturesand Epidural Catheterisations: Systematic Review and Meta-Analysis. BMJ, 346, f1720. https://doi.org/10.1136/bmj.f1720

[15] Gambling, D.R. (2011) Lumbar Ultrasound: Useful Gadget or Time-Consuming Gimmick? International Journal of Obstetric Anesthesia, 20, 318-320. https://doi.org/10.1016/j.ijoa.2010.10.001

[16] Tiouririne, M., Dixon, A.J., Mauldin, F.W., et al. (2017) Imaging Performance of a Handheld Ultrasound System with Real-Time Computer-Aided Detection of Lumbar Spine Anatomy: A Feasibility Study. Investigative Radiology, 52, 447-455. https://doi.org/10.1097/RLI.0000000000000361

[17] Deacon, A.J., Melhuishi, N.S. and Terblanche, N.C. (2014) CUSUM Method for Construction of Trainee Spinal Ultrasound Learning Curves Following Standardised Teaching. Anaesthesia and Intensive Care, 42, 480-486.

[18] Margarido, C.B., Arzola, C., Balki, M., et al. (2010) Anesthesiologists' Learning Curves for Ultrasound Assessment of the Lumbar Spine. Canadian Journal of Anesthesia, 57, 120-126. https://doi.org/10.1007/s12630-009-9219-2 
[19] Halpern, S.H., Banerjee, A., Stocche, R. and Glanc, P. (2010) The Use of Ultrasound for Lumbar Spinous Process Identification. A Pilot Study. Canadian Journal of Anesthesia, 57, 817-822. https://doi.org/10.1007/s12630-010-9337-x

[20] Karmakar, M.K., Li, X., Ho, A.M., et al. (2009) Real-Time Ultrasound-Guided Paramedian Epiduralaccess: Evaluation of a Novel In-Plane Technique. British Journal of Anaesthesia, 102, 845-854. https://doi.org/10.1093/bja/aep079

[21] Vallejo, M.C., Phelps, A.L., Singh, S., et al. (2010) Ultrasound Decreases the Failed Laborepidural Rate in Resident Trainees. International Journal of Obstetric Anesthesia, 19, 373-378. https://doi.org/10.1016/j.ijoa.2010.04.002 